\title{
Estabilidade de repolho minimamente processado sob diferentes sistemas de embalagem
}

\author{
Storage of minimally processed cabbage in differents packaging systems \\ Maria Madalena RINALDI ${ }^{1 *}$, Benedito Carlos BENEDETTI ${ }^{2}$, \\ Claire Isabel Grígoli de Luca SARANTÓPOULOS ${ }^{3}$, Celso Luiz MORETTI ${ }^{4}$
}

\begin{abstract}
Resumo
Avaliou-se a estabilidade de repolho minimamente processado acondicionado em embalagens de polietileno de baixa densidade com atmosfera modificada ativa e passiva, e em bandejas de poliestireno expandido revestidas com filme de policloreto de vinila. O produto foi armazenado por 16 dias em câmara frigorífica na temperatura de $5 \pm 1{ }^{\circ} \mathrm{C}$ e $95 \pm 5 \%$ de umidade relativa, e em um balcão refrigerado similar aos utilizados em lojas de conveniência, com o intuito de representar os locais de comercialização no varejo, com temperatura média de $2,7 \pm 3,3^{\circ} \mathrm{C}$. Analisou-se a concentração de oxigênio e dióxido de carbono no interior das embalagens, e no repolho minimamente processado o incremento no escurecimento, luminosidade, atividade da polifenoloxidase e peroxidase, $\mathrm{pH}$, acidez titulável, sólidos solúveis, perda de massa fresca e teor de ácido ascórbico. A estabilidade do repolho minimamente processado mostrou-se superior na temperatura de $5{ }^{\circ} \mathrm{C}$ quando comparada ao armazenamento no balcão refrigerado. A atmosfera modificada ativa não foi efetiva no aumento da vida útil do repolho minimamente processado quando comparada aos outros tratamentos. A embalagem de PEBD foi a mais adequada ao acondicionamento do repolho minimamente processado. No entanto, a embalagem de PVC também apresentou resultados satisfatórios.

Palavras-chave: Brassica oleracea var. capitata; atmosfera modificada ativa; atmosfera modificada passiva; vida útil.
\end{abstract}

\begin{abstract}
Stability of fresh-cut cabbage packed in low density polyethylene under active modified atmosphere and in expanded polyestyrene trays wrapped with PVC was evaluated. The fresh-cut product was stored for 16 days in a cold room at $5 \pm 1{ }^{\circ} \mathrm{C}$ and $95 \pm 5 \% \mathrm{RH}$ and in a refrigerated display case similar to those found in grocery stores. The following variables were analyzed: content of oxygen and carbon dioxide inside the package headspace; browning, luminosity, polyphenoloxidase and peroxidase activities, $\mathrm{pH}$, titratable acidity; soluble solids, fresh mass loss, and ascorbic acid content. The stability of fresh-cut cabbage was higher at the temperature of $5{ }^{\circ} \mathrm{C}$ compared to those stored in the refrigerated displays. The active modified atmosphere was not effective in extending the shelf life of fresh-cut cabbage compared to other treatments. LDPE packaging was more adequate for fresh-cut cabbage storage. However, PVC wrapping also presented satisfactory effects.

Keywords: Brassica oleracea var. capitata; active modified atmosphere; passive modified atmosphere; shelf life.
\end{abstract}

\section{Introdução}

A modificação nos hábitos alimentares da população brasileira em busca da melhoria na qualidade de vida e longevidade tem incentivado a procura por alimentos saudáveis, de excelente qualidade sensorial e garantia de sanidade, aumentando o consumo de hortaliças e frutas frescas (DURIGAN, 2004). O processamento mínimo objetiva suprir essas necessidades, disponibilizando produtos frescos, limpos, convenientes, preparados e adequados para o consumo em menor tempo (CANTWELL; SUSLOW, 2002). Consequentemente, ocorre a agregação de valor aos produtos, maior aproveitamento da produção, redução das perdas pós-colheita e maior eficiência no manejo de resíduos (MATTIUZ, 2004).

Apesar das vantagens apresentadas por estes produtos, há problemas em sua conservação. A utilização de atmosfera modificada e redução da temperatura de armazenamento aumentam a vida útil de diversas hortaliças e frutas minimamente processadas (RINALDI; BENEDETTI, 2004; TELES et al., 2005). Atmosferas com 2 a $8 \%$ de $\mathrm{O}_{2}$ e 5 a $15 \%$ de $\mathrm{CO}_{2}$ têm potencial para aumentar a vida útil e viabilizar a comercialização destes produtos. Porém, para cada vegetal existe uma atmosfera específica que maximiza sua durabilidade (CANTWELL; SUSLOW, 2002). Para o repolho minimamente processado, a temperatura indicada para a conservação varia de 0 a $5{ }^{\circ} \mathrm{C}$ (CANTWELL; SUSLOW, 2002) e a ótima concentração de $\mathrm{O}_{2}$ está entre 2,2 e $4,3 \%$ (KAWANO, 1984). Por outro lado, o repolho inteiro apresenta excelentes condições de armazenamento e transporte na temperatura de 0 a $5{ }^{\circ} \mathrm{C}$ e 2 a $3 \%$ de $\mathrm{O}_{2}$ e 3 a $6 \%$ de $\mathrm{CO}_{2}$ (KADER; SINGH; MANNAPPERUMA, 1998). Entretanto,

\footnotetext{
Recebido para publicação em 18/9/2007

Aceito para publicação em 7/3/2008 (002862)

Centro de Pesquisa Agropecuária do Cerrado - EMBRAPA Cerrados, BR 020 km 18, Rodovia Brasília/Fortaleza, CP 08223, CEP 73310-970, Brasília - DF, Brasil,

E-mail: maria.rinaldi@cpac.embrapa.br

${ }^{2}$ Faculdade de Engenharia Agrícola - FEAGRI, Universidade Estadual de Campinas - UNICAMP, Campinas - SP, Brasil

${ }^{3}$ Centro de Ensino e Tecnologia de Araçatuba - CETEA, Instituto de Tecnologia de Alimentos - ITAL, Campinas - SP, Brasil

${ }^{4}$ Centro Nacional de Pesquisa de Hortaliças - CNPH, EMBRAPA, Brasília - DF, Brasil

${ }^{*}$ A quem a correspondência deve ser enviada
} 
Gorny (2001) recomenda a mesma faixa de temperatura e 5 a $7,5 \%$ de $\mathrm{O}_{2}$ e $15 \%$ de $\mathrm{CO}_{2}$.

Diante do exposto, este trabalho objetivou avaliar a estabilidade do repolho minimamente processado, acondicionado em embalagens com atmosfera modificada passiva e ativa, durante o armazenamento em câmara frigorífica a $5{ }^{\circ} \mathrm{C}$ e em balcão refrigerado.

\section{Material e métodos}

Repolhos (Brassica oleracea var. capitata), variedade Sagitarius, foram obtidos de plantação comercial localizada no município de Morungaba/SP com 90 dias de campo, sendo selecionados, lavados em água corrente e resfriados em câmara fria $\left(5 \pm 2{ }^{\circ} \mathrm{C}\right.$ e $95 \pm 5 \%$ de umidade relativa). $\mathrm{O}$ processamento mínimo consistiu em corte manual das cabeças de repolho em quatro partes, retirada das partes internas (talos), fatiamento em tiras $(3 \mathrm{~mm}) \mathrm{em}$ processador industrial de alimentos marca SKYMSEN, modelo PA-7L; enxágue (3 minutos) em água tratada; imersão (10 minutos) em solução sanitizante de hipoclorito de sódio $\left(150 \mathrm{mg} \mathrm{L}^{-1}\right.$ de cloro ativo); enxágue (5 minutos) com solução $\left(5 \mathrm{mg} \mathrm{L}^{-1}\right)$ do mesmo sanitizante e centrifugação ( 5 minutos) em centrífuga doméstica com velocidade angular média de $800 \mathrm{~g}$. A temperatura da água de lavagem, sanitização e enxágues foi de $5 \pm 2{ }^{\circ} \mathrm{C}$ e a temperatura do ambiente foi mantida a $15 \pm 3{ }^{\circ} \mathrm{C}$. Adotaram-se Boas Práticas de Fabricação (VANETTI, 2004).

O repolho minimamente processado $(300 \mathrm{~g})$ foi acondicionado em bandejas de poliestireno expandido $(210 \times 140 \times 20 \mathrm{~mm})$ revestidas com filme esticável de policloreto de vinila (PVC), $20 \mu \mathrm{m}$ de espessura, com taxa de permeabilidade ao oxigênio de $8212 \mathrm{~cm}^{3} \mathrm{~m}^{-2} \mathrm{dia}^{-1}$, dióxido de carbono de $61260 \mathrm{~cm}^{3} \mathrm{~m}^{-2} \mathrm{dia}^{-1} \mathrm{e}$ área de permeação de $730 \mathrm{~cm}^{2}$. O fechamento desta embalagem foi realizado por aquecimento na parte inferior da bandeja, em uma seladora marca BARBI, modelo B-500. Utilizou-se, também, polietileno de baixa densidade (PEBD) $(250 \times 350 \mathrm{~mm}), 32 \mu \mathrm{m}$ de espessura, sem perfurações, com taxa de permeabilidade ao oxigênio de $6.169 \mathrm{~cm}^{3} \mathrm{~m}^{-2} \mathrm{dia}^{-1}$, área de permeação de $1.750 \mathrm{~cm}^{2}$ e taxa de permeabilidade ao vapor de água de $14,2 \mathrm{~g}$ de água $\mathrm{m}^{-2} \mathrm{dia}^{-1}$. Esta embalagem foi utilizada para gerar uma atmosfera modificada passiva sendo fechada em uma seladora marca BARBI, modelo TI 400. Nesta embalagem, testou-se, também, a injeção de uma atmosfera modificada $\left(2 \% \mathrm{O}_{2} ; 6,5 \%\right.$ $\mathrm{CO}_{2} ; 91,5 \% \mathrm{~N}_{2}$ ), utilizando-se uma seladora marca SELOVAC, modelo 200B. A estimativa da permeabilidade ao oxigênio $\left(7.700 \mathrm{~cm}^{3} \mathrm{~m}^{-2} \mathrm{dia}^{-1}\right)$ e ao dióxido de carbono $\left(42.000 \mathrm{~cm}^{3} \mathrm{~m}^{-2} \mathrm{dia}^{-1}\right)$ do filme, necessária para o acondicionamento do repolho minimamente processado, foi baseada nas equações apresentadas por Cameron et al. (1995) e Oliveira et al. (1996).

As embalagens contendo o produto foram armazenadas durante 16 dias em câmara frigorífica a $5 \pm 1{ }^{\circ} \mathrm{C}$ e $95 \pm 5 \%$ de umidade relativa, e em um balcão tipo expositor refrigerado, similar aos utilizados em lojas de conveniência, com porta de vidro reto, marca GELOPAR, modelo GERC 210-R1, com o intuito de simular os locais de comercialização no varejo. $\mathrm{O}$ termostato do equipamento foi ajustado na posição 1 , que, de acordo com as orientações do fabricante, deveria proporcionar uma temperatura média no interior do balcão em torno de $10^{\circ} \mathrm{C} \pm 1{ }^{\circ} \mathrm{C}$. Além disso, 6 termopares previamente calibrados foram colocados em seu interior sendo 3 na prateleira superior ( 1 à esquerda, 1 ao centro e 1 à direita) e outros 3 na prateleira inferior, nas mesmas posições. Os dados de temperatura foram coletados por um Data logger marca ECO-série 5000, modelo 5100, com leituras a cada 5 minutos.

Após o processamento mínimo do repolho e nos dias 4, 7, 9, 11, 14 e 16 de armazenamento, analisou-se a concentração de oxigênio e dióxido de carbono, em porcentagem, no interior das embalagens, com um analisador de gás (Head Space Analyser, MOCON), previamente calibrado com gases padrões. Luminosidade em espectrofotômetro de bancada marca Hunter Lab, modelo Color Quest II, taxa de escurecimento $\left(\mathrm{IE}=\sqrt{\left(\mathrm{L}-\mathrm{L}_{0}\right)^{2}+\left(\mathrm{a}-\mathrm{a}_{0}\right)^{2}+\left(\mathrm{b}-\mathrm{b}_{0}\right)^{2}}\right)$, atividade da enzima polifenoloxidase (PPO) (ALMEIDA; NOGUEIRA, 1995) atividade da enzima peroxidase (POD) (KHAN; ROBINSON, 1994), e pH, ácido ascórbico, acidez titulável e sólidos solúveis de acordo com metodologias do Instituto Adolfo Lutz (1985). A perda de massa fresca foi determinada utilizando-se balança digital marca Gehaka, modelo BG 2000 com precisão de $0,01 \mathrm{~g}$.

O delineamento experimental adotado foi o inteiramente casualizado, com três repetições para cada tratamento (PVC $5^{\circ} \mathrm{C}$ e balcão refrigerado, PEBDpassiva $5^{\circ} \mathrm{C}$ e balcão refrigerado e PEBDativa $5{ }^{\circ} \mathrm{C}$ e balcão refrigerado), em que estudou-se a interação entre os fatores: condições de armazenamento $\left(5^{\circ} \mathrm{C} \pm 1{ }^{\circ} \mathrm{C}\right.$ e balcão refrigerado $\left(2,7^{\circ} \mathrm{C} \pm 3,3^{\circ} \mathrm{C}\right)$, embalagem (PVC, PEBDativa e PEBDpassiva) e tempo de armazenamento $(0,4,7,9,11,14$ e 16 dias). A análise estatística dos dados foi realizada com o auxílio do software ESTAT ([19--]).

\section{Resultados e discussão}

\subsection{Temperatura no balcão expositor refrigerado}

A temperatura média no interior do balcão expositor durante os 16 dias de armazenamento do repolho minimamente processado foi de $2,7^{\circ} \mathrm{C} \pm 3,3^{\circ} \mathrm{C}$, ocorrendo temperatura negativa em pequenos períodos de análise, não afetando a vida útil do produto. O coeficiente de variação foi de $122,0 \%$. O objetivo inicial era manter o produto submetido à temperatura média de $10{ }^{\circ} \mathrm{C}$, no entanto, o equipamento utilizado não correspondeu ao desejado, produzindo uma temperatura média inferior, inclusive, à câmara refrigerada, sendo este resultado positivo para o produto que foi mantido em temperatura mais adequada para o seu armazenamento.

\subsection{Composição gasosa no interior das embalagens}

A embalagem de PEBDativa não apresentou alteração significativa na porcentagem de oxigênio até o $11^{\circ}$ dia de armazenamento nas duas condições de armazenamento (Tabela 1), com os valores de oxigênio mantendo-se na faixa recomendada para o armazenamento do repolho (KAWANO et al., 1984). A permeabilidade a gases desta embalagem e a taxa respiratória do produto levaram à menor concentração de oxigênio no interior da embalagem (SARANTÓPOULOS et al., 2003). A partir do 
$11^{\circ}$ dia de armazenamento ocorreu aumento significativo na concentração de oxigênio, atingindo $12 \%$ no $14^{\circ}$ dia.

As embalagens de PEBDpassiva e PVC apresentaram redução acentuada na concentração de oxigênio até o $4^{\circ}$ dia, chegando a 5,82\% na embalagem de PEBDpassiva em balcão refrigerado (Tabela 1). O contato entre enzimas e substratos, após a desestruturação dos sistemas de membranas celulares causado pelo corte do produto, acelera o processo respiratório (SAKR, 1997). A partir do $4^{\circ}$ dia, os valores de oxigênio oscilaram entre 3 e $12 \%$ para a embalagem de PEBDpassiva e entre 5 e 13\% para a PVC, encontrando-se, na maioria dos períodos de análise, com concentração inferior à atmosférica e, superior à faixa recomendada para o armazenamento do repolho minimamente processado, podendo ter influenciado na taxa respiratória do produto (ZAGORY, 1999).

Com exceção do $14^{\circ}$ dia de armazenamento, a embalagem de PVC sempre apresentou a maior concentração de oxigênio, provavelmente, devido à maior taxa de permeabilidade a gases dessa embalagem, chegando a um mínimo de $5 \%$ no $7^{\circ}$ dia de armazenamento. As embalagens mantidas em balcão refrigerado, com algumas exceções, sempre apresentaram menor concentração de oxigênio, provavelmente, devido à maior taxa respiratória do produto.

De maneira geral, as embalagens mantidas no balcão refrigerado apresentaram maior concentração de dióxido de carbono que a $5{ }^{\circ} \mathrm{C}$ (Tabela 1$)$, provavelmente devido à maior taxa respiratória induzida pela oscilação de temperatura e acúmulo deste gás no interior da embalagem. A embalagem de PEBDativa não manteve a concentração inicial de $6,5 \%$ de dióxido de carbono chegando a $5 \%$ no $4^{\circ}$ dia. No entanto, este tratamento apresentou a maior concentração deste gás durante

Tabela 1. Valores médios de oxigênio e dióxido de carbono, na atmosfera interna de embalagens contendo repolho minimamente processado armazenadas durante 16 dias em câmara frigorífica a $5 \pm 1{ }^{\circ} \mathrm{C}$ e em balcão refrigerado $\left(2,7 \pm 3,3^{\circ} \mathrm{C}\right)$.

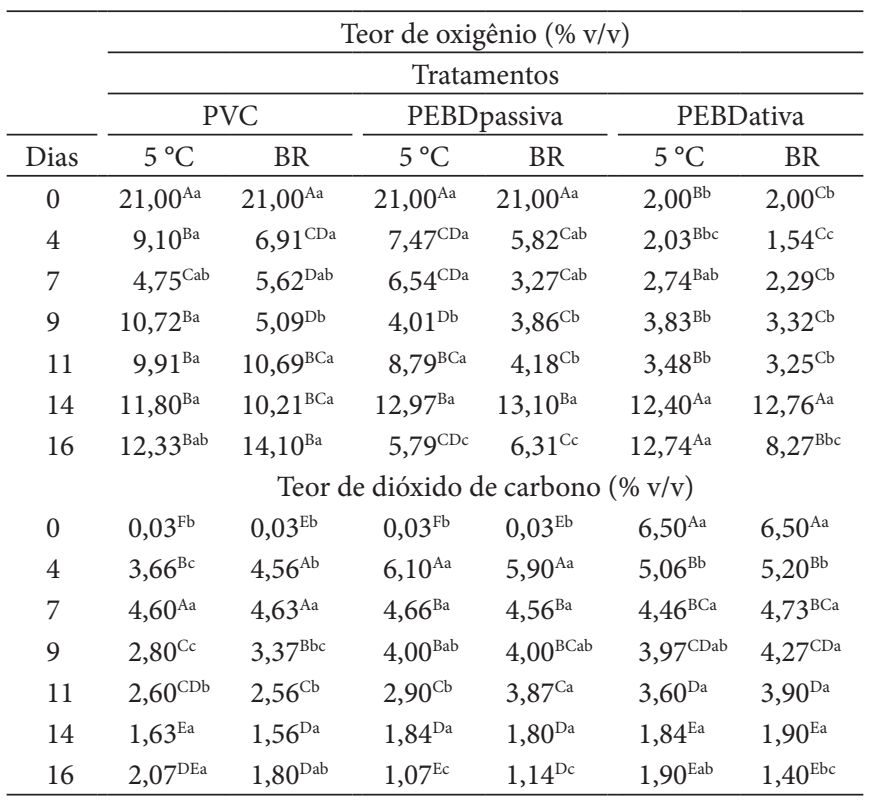

Médias seguidas por mesma letra maiúscula na coluna e minúscula na linha não diferem entre si pelo teste de Tukey $(\mathrm{p} \leq 0,01)$; e BR - armazenamento em balcão refrigerado. todo o armazenamento, sendo que a maior atividade respiratória pode ter compensado, em parte, o dióxido de carbono perdido através da embalagem (ZAGORY, 1995). De maneira geral, não ocorreu estabilidade na concentração de $\mathrm{CO}_{2}$, durante todo $\mathrm{o}$ armazenamento, independente da embalagem, atmosfera e condição de armazenamento não atingindo uma atmosfera de equilíbrio na embalagem.

\subsection{Incremento no escurecimento e luminosidade do repolho minimamente processado}

Os produtos acondicionados nas embalagens de PVC e PEBDpassiva mantidas em balcão refrigerado apresentaram o maior grau de escurecimento (Tabela 2) nos primeiros quatro dias de armazenamento, chegando a um valor máximo de 5,5. Os demais tratamentos apresentaram escurecimento acentuado até $07^{\circ}$ dia de armazenamento. A partir desse período, os valores oscilaram entre 2,5 e 6,3 para todos os tratamentos.

Os valores de luminosidade oscilaram entre 81,52 , logo após o processamento, e 74,89 , no $16^{\circ}$ dia, representando uma perda total de $8,13 \%$ durante o armazenamento. A maior perda ocorreu nos primeiros quatro dias, correspondendo ao maior incremento no escurecimento (Tabela 2).

\subsection{Atividade enzimática}

O valor inicial de atividade da polifenoloxidase foi de 57,60 UAE $\mathrm{min}^{-1} \mathrm{~g}^{-1} \mathrm{MF}$ (Tabela 3). Até o $4^{\circ}$ dia de armazenamento o repolho minimamente processado submetido a todos os tratamentos apresentou aumento significativo no teor de polifenoloxidase, correspondendo à maior diminuição na

Tabela 2. Valores médios de incremento de escurecimento e luminosidade em repolho minimamente processado, acondicionado em embalagens armazenadas durante 16 dias em câmara frigorífica a $5 \pm 1^{\circ} \mathrm{C}$ e em balcão refrigerado $\left(2,7 \pm 3,3^{\circ} \mathrm{C}\right)$.

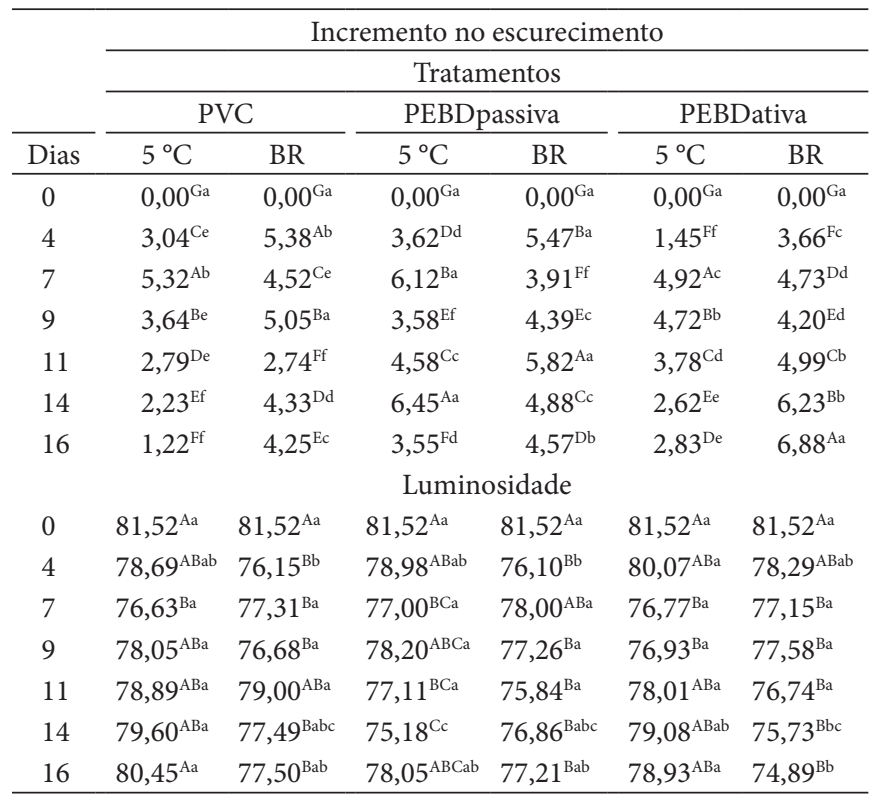

Médias seguidas por mesma letra maiúscula na coluna e minúscula na linha não diferem entre si pelo teste de Tukey $(\mathrm{p} \leq 0,01)$; e BR - armazenamento em balcão refrigerado. 
luminosidade e maior incremento no escurecimento (Tabela 2). Tal fenômeno foi menos acentuado no produto acondicionado na embalagem de PEBDpassiva a $5{ }^{\circ} \mathrm{C}$ e PVC. Após esse período os valores oscilaram entre $31,15 \mathrm{UAE} \mathrm{min}^{-1} \mathrm{~g}^{-1} \mathrm{MF}$ e 314,40 UAE min $^{-1} \mathrm{~g}^{-1} \mathrm{MF}$. De maneira geral, o repolho minimamente processado não apresentou comportamento linear quanto à atividade da enzima polifenoloxidase, correspondendo aos valores de luminosidade e incremento no escurecimento. O escurecimento em tecidos vegetais pode ocorrer pela degradação do ácido ascórbico (KLEIN, 1987), reações oxidativas (SCHLIMME, 1995), pH do produto (ARAÚJO, 1999), concentração de oxigênio na embalagem (WHITAKER; LEE, 1995), nível de injúria mecânica e exposição ao etileno (YANO; SAIJO, 1987) e o tipo de polifenoloxidase presente no vegetal analisado (GOUPY, 1995). O repolho minimamente processado apresentou valores entre $350 \mathrm{UAE}$ min $^{-1} \mathrm{~g}^{-1} \mathrm{MF}$ e $800 \mathrm{UAE} \mathrm{min}^{-1} \mathrm{~g}^{-1} \mathrm{MF}$, sendo significativamente superiores aos observados neste trabalho (SILVA, 2000). Em 25 cultivares de repolho cortados, oito apresentaram leve escurecimento e duas com escurecimento mínimo após 24 horas de armazenamento a $20^{\circ} \mathrm{C}$ (YANO; SAIJO, 1987).

A atividade da enzima peroxidase (Tabela 3 ) oscilou entre 139,45 UAE min $^{-1} \mathrm{~g}^{-1} \mathrm{MF}$ e 713,55 UAE $\mathrm{min}^{-1} \mathrm{~g}^{-1} \mathrm{MF}$, com maior variação e atividade que a polifenoloxidase, também não apresentando linearidade durante $\mathrm{o}$ armazenamento. A atividade de peroxidase foi significativa durante o armazenamento do repolho minimamente processado (NAWA et al., 1987). O pH do repolho minimamente processado contempla a faixa ótima para a atividade da peroxidase (ARAÚJO, 1999; FUJITA, 1995). A maior atividade da peroxidase corresponde ao maior incremento no escurecimento, diminuição da luminosidade e ácido ascórbico.

\section{5 pH e acidez titulável}

$\mathrm{O}$ valor inicial de $\mathrm{pH}$ foi de 5,44, chegando a um máximo de 6,52 com diferença significativa durante os
16 dias de armazenamento (Tabela 4), sendo classificado como um produto não ácido e mais propício à presença $\mathrm{e}$ desenvolvimento de microrganismos patogênicos (CHITARRA; CHITARRA, 1990). Ocorreu aumento do $\mathrm{pH}$ até o $9^{\circ}$ dia, e correspondente redução da acidez (Tabela 4), e o repolho acondicionado na embalagem de PEBDpassiva apresentou, durante todo o período, valores de $\mathrm{pH}$ superiores aos demais

Tabela 4. Valores médios de $\mathrm{pH}$ e acidez titulável em repolho minimamente processado, acondicionado em embalagens armazenadas durante 16 dias em câmara frigorífica a $5 \pm 1{ }^{\circ} \mathrm{C}$ e em balcão refrigerado $\left(2,7 \pm 3,3^{\circ} \mathrm{C}\right)$.

\begin{tabular}{|c|c|c|c|c|c|c|}
\hline \multirow[b]{4}{*}{ Dias } & \multicolumn{6}{|c|}{$\mathrm{pH}$} \\
\hline & \multicolumn{6}{|c|}{ Tratamentos } \\
\hline & \multicolumn{2}{|c|}{ PVC } & \multicolumn{2}{|c|}{ PEBDpassiva } & \multicolumn{2}{|c|}{ PEBDativa } \\
\hline & $5^{\circ} \mathrm{C}$ & $\mathrm{BR}$ & $5^{\circ} \mathrm{C}$ & BR & $5^{\circ} \mathrm{C}$ & BR \\
\hline 0 & $5,44^{\mathrm{Da}}$ & $5,44^{\mathrm{Ca}}$ & $5,44^{\mathrm{Da}}$ & $5,44^{\mathrm{Ea}}$ & $5,44^{\mathrm{Da}}$ & $5,44^{\mathrm{Ea}}$ \\
\hline 4 & $5,66^{\mathrm{Cc}}$ & $5,69^{\mathrm{Bc}}$ & $6,16^{\mathrm{Ca}}$ & $5,93^{\mathrm{Db}}$ & $5,78^{\mathrm{Cbc}}$ & $5,76^{\mathrm{Dbc}}$ \\
\hline 7 & $6,27^{\mathrm{ABa}}$ & $6,27^{\mathrm{Aa}}$ & $6,37^{\mathrm{ABCa}}$ & $6,23^{\mathrm{Ca}}$ & $6,19^{\mathrm{Ba}}$ & $6,19^{\mathrm{Ca}}$ \\
\hline 9 & $6,37^{\mathrm{Aa}}$ & $6,36^{\mathrm{Aa}}$ & $6,43^{\mathrm{Aa}}$ & $6,46^{\mathrm{ABa}}$ & $6,34^{\mathrm{ABa}}$ & $6,40^{\mathrm{ABa}}$ \\
\hline 11 & $6,25^{\mathrm{ABb}}$ & $6,35^{\text {Aab }}$ & $6,37^{\mathrm{ABab}}$ & $6,47^{\mathrm{ABa}}$ & $6,35^{\mathrm{ABab}}$ & $6,26^{\mathrm{ABCb}}$ \\
\hline 14 & $6,25^{\mathrm{ABb}}$ & $6,30^{\mathrm{Ab}}$ & $6,42^{\mathrm{Aab}}$ & $6,52^{\mathrm{Aa}}$ & $6,43^{\mathrm{Aab}}$ & $6,43^{\mathrm{Aab}}$ \\
\hline \multirow[t]{2}{*}{16} & $6,15^{\mathrm{Bb}}$ & $6,21^{\mathrm{Aab}}$ & $6,19^{\mathrm{BCab}}$ & $6,26^{\mathrm{BCab}}$ & $6,35^{\mathrm{ABa}}$ & $6,20^{\mathrm{BCab}}$ \\
\hline & \multicolumn{6}{|c|}{ Acidez titulável (meq ácido cítrico $\mathrm{kg}^{-1} \mathrm{MF}$ ) } \\
\hline 0 & $0,91^{\mathrm{Aa}}$ & $0,91^{\mathrm{Aa}}$ & $0,91^{\text {Aa }}$ & $0,91^{\mathrm{Aa}}$ & $0,91^{\mathrm{Aa}}$ & $0,91^{\mathrm{Aa}}$ \\
\hline 4 & $0,66^{\mathrm{Ba}}$ & $0,59^{\mathrm{Bab}}$ & $0,42^{\mathrm{BCc}}$ & $0,55^{\mathrm{Bb}}$ & $0,57^{\mathrm{Bb}}$ & $0,57^{\mathrm{Bb}}$ \\
\hline 7 & $0,53^{\mathrm{Ca}}$ & $0,48^{\mathrm{Ca}}$ & $0,39^{\mathrm{Cb}}$ & $0,47^{\mathrm{BCDa}}$ & $0,51^{\mathrm{BCa}}$ & $0,48^{\mathrm{CDa}}$ \\
\hline 9 & $0,50^{\mathrm{Ca}}$ & $0,49^{\mathrm{Ca}}$ & $0,43^{\mathrm{BCa}}$ & $0,43^{\mathrm{CDa}}$ & $0,49^{\mathrm{BCa}}$ & $0,45^{\mathrm{CDEa}}$ \\
\hline 11 & $0,46^{\mathrm{Cab}}$ & $0,41^{\mathrm{Cab}}$ & $0,38^{\mathrm{Cb}}$ & $0,39^{\text {Dab }}$ & $0,47^{\mathrm{Ca}}$ & $0,42^{\text {DEab }}$ \\
\hline 14 & $0,46^{\mathrm{Cab}}$ & $0,47^{\mathrm{Ca}}$ & $0,36^{\mathrm{Cc}}$ & $0,39^{\mathrm{Dabc}}$ & $0,38^{\mathrm{Dbc}}$ & $0,37^{\mathrm{Ec}}$ \\
\hline 16 & $0,53^{\mathrm{Ca}}$ & $0,48^{\mathrm{Ca}}$ & $0,49^{\mathrm{Ba}}$ & $0,49^{\mathrm{BCa}}$ & $0,45^{\mathrm{CDa}}$ & $0,51^{\mathrm{BCa}}$ \\
\hline
\end{tabular}

Médias seguidas por mesma letra maiúscula na coluna e minúscula na linha não diferem entre si pelo teste de Tukey $(\mathrm{p} \leq 0,01)$; e BR - armazenamento em balcão refrigerado.

Tabela 3. Valores médios de atividade das enzimas polifenoloxidase e peroxidase em repolho minimamente processado, acondicionado em embalagens armazenadas durante 16 dias em câmara frigorífica a $5 \pm 1{ }^{\circ} \mathrm{C}$ e em balcão refrigerado $\left(2,7 \pm 3,3^{\circ} \mathrm{C}\right)$.

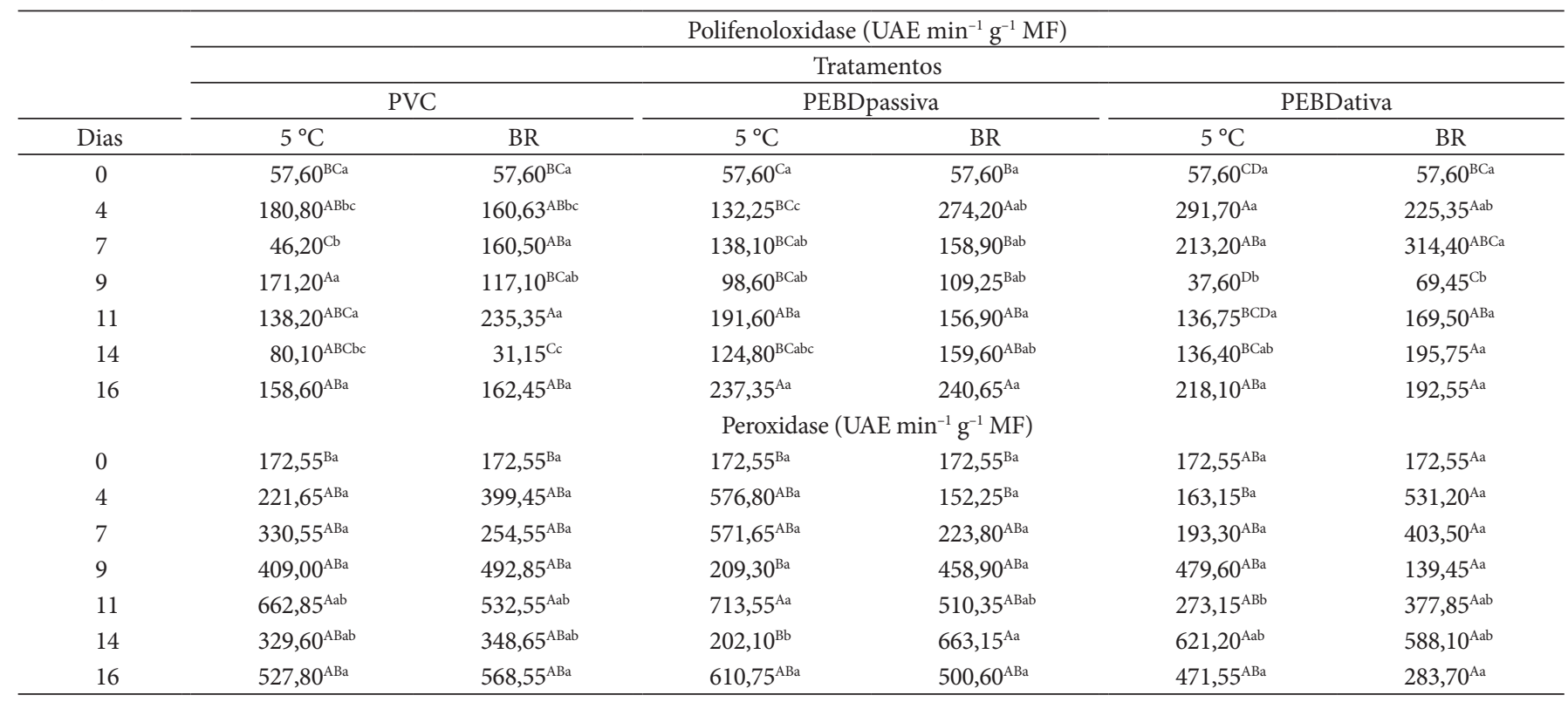

Médias seguidas por mesma letra maiúscula na coluna e minúscula na linha não diferem entre si pelo teste de Tukey (p $\leq 0,01)$; e BR - armazenamento em balcão refrigerado. 
tratamentos. O repolho minimamente processado embalado em filmes de polietileno de baixa densidade e polipropileno mantidos a $5 \pm 1{ }^{\circ} \mathrm{C}$ também apresentou valores iniciais de pH em torno de 5,5 (SILVA, 2000). FANTUZZI et al. (2004) e GÓMEZ-LÓPEZ et al. (2007) também observaram aumento significativo nos valores de $\mathrm{pH}$ de repolho minimamente processado durante o armazenamento, sendo que pode estar relacionado com a resposta do tecido ao tentar neutralizar a acidez gerada pelo dióxido de carbono (KADER, 1986), em que a concentração deve ser no mínimo $5 \%$ para que ocorra influência no pH intracelular (WILEY, 1994). O comportamento do $\mathrm{pH}$ nos produtos minimamente processados pode ser devido ao aumento na população de microrganismos durante o armazenamento (MARTH, 1998).

Ocorreu redução significativa na acidez titulável do repolho minimamente processado em todos os tratamentos, sendo mais acentuada entre o início do armazenamento e o $4^{\circ}$ dia, no produto acondicionado na embalagem de PEBDpassiva na temperatura de $5^{\circ} \mathrm{C}$ (Tabela 4). A partir do $7^{\circ}$ dia, os valores oscilaram entre 0,40 meq de ácido cítrico $\mathrm{kg}^{-1} \mathrm{MF}$ e 0,55 meq de ácido cítrico $\mathrm{kg}^{-1} \mathrm{MF}$. De maneira geral, durante todo o armazenamento, o produto acondicionado na embalagem de PVC apresentou os maiores valores de acidez titulável resultando em menor perda de ácidos orgânicos, não correspondendo ao esperado, pois esta embalagem, na maioria dos períodos de análise, apresentou concentração de oxigênio superior às demais embalagens. Rinaldi et al. (2005) observaram redução significativa na acidez de repolho minimamente processado acondicionado em embalagens de PVC, $12 \mu \mathrm{m}$, e PET armazenadas nas temperaturas de 0,5 e $10^{\circ} \mathrm{C}$, sendo que o repolho mantido na temperatura de $0{ }^{\circ} \mathrm{C}$ apresentou maior acidez no final do armazenamento. Os ácidos orgânicos são encontrados nos vacúolos das células na forma livre e combinados com sais, ésteres e glicosídeos, como fonte importante de energia para os vegetais durante o processo de maturação, sofrendo oxidação no ciclo de Krebs (KLUGE et al., 2002).

\subsection{Sólidos solúveis, perda de massa fresca e, ácido ascórbico}

Não ocorreu redução significativa nos sólidos solúveis e perda de massa fresca no repolho minimamente processado durante o armazenamento (Tabela 5), correspondendo aos resultados obtidos por Silva (2000). Os valores de sólidos solúveis oscilaram entre 3,25 e $4,25^{\circ} \mathrm{Brix}$. A porcentagem de perda de massa fresca atingiu o valor máximo de $0,46 \%$, não sendo significativa.

Durante os 16 dias de armazenamento o repolho minimamente processado apresentou uma perda total de 23,79\% de ácido ascórbico (Tabela 5). De maneira geral, com exceção de algumas diferenças entre os tratamentos, provavelmente devido à variabilidade das amostras, ocorreu redução de ácido ascórbico até o $9^{\circ}$ dia de armazenamento, a partir do qual houve aumento nos valores igualando-se aos iniciais. Na embalagem de PVC o repolho apresentou redução menos acentuada durante esse período. O ácido ascórbico
Tabela 5. Valores médios de sólidos solúveis, perda de massa fresca e, ácido ascórbico em repolho minimamente processado, acondicionado em embalagens armazenadas durante 16 dias em câmara frigorífica a $5 \pm 1{ }^{\circ} \mathrm{C}$ e em balcão refrigerado $\left(2,7 \pm 3,3^{\circ} \mathrm{C}\right)$.

\begin{tabular}{|c|c|c|c|c|c|c|}
\hline \multirow[b]{4}{*}{ Dias } & \multicolumn{6}{|c|}{ Sólidos solúveis ( $\left.{ }^{\circ} \mathrm{Brix}\right)$} \\
\hline & \multicolumn{6}{|c|}{ Tratamentos } \\
\hline & \multicolumn{2}{|c|}{ PVC } & \multicolumn{2}{|c|}{ PEBDpassiva } & \multicolumn{2}{|c|}{ PEBDativa } \\
\hline & $5^{\circ} \mathrm{C}$ & BR & $5^{\circ} \mathrm{C}$ & $\mathrm{BR}$ & $5{ }^{\circ} \mathrm{C}$ & $\mathrm{BR}$ \\
\hline 0 & $4,25^{\mathrm{Aa}}$ & $4,25^{\mathrm{Aa}}$ & $4,25^{\mathrm{Aa}}$ & $4,25^{\mathrm{Aa}}$ & $4,25^{\mathrm{Aa}}$ & $4,25^{\mathrm{Aa}}$ \\
\hline 4 & $3,83^{\mathrm{Aa}}$ & $3,58^{\mathrm{Aa}}$ & $3,58^{\mathrm{Aa}}$ & $3,75^{\mathrm{Aa}}$ & $3,83^{\mathrm{Aa}}$ & $3,75^{\mathrm{Aa}}$ \\
\hline 7 & $3,92^{\mathrm{Aa}}$ & $3,75^{\mathrm{Aa}}$ & $3,83^{\mathrm{Aa}}$ & $3,83^{\mathrm{Aa}}$ & $3,75^{\mathrm{Aa}}$ & $3,75^{\mathrm{Aa}}$ \\
\hline 9 & $3,75^{\mathrm{Aa}}$ & $3,65^{\mathrm{Aa}}$ & $3,25^{\mathrm{Aa}}$ & $3,34^{\mathrm{Aa}}$ & $3,58^{\mathrm{Aa}}$ & $3,34^{\mathrm{Aa}}$ \\
\hline 11 & $4,17^{\mathrm{Aa}}$ & $3,75^{\mathrm{Aa}}$ & $3,67^{\mathrm{Aa}}$ & $3,50^{\mathrm{Aa}}$ & $3,75^{\mathrm{Aa}}$ & $3,58^{\mathrm{Aa}}$ \\
\hline 14 & $3,75^{\mathrm{Aa}}$ & $3,75^{\mathrm{Aa}}$ & $3,34^{\mathrm{Aa}}$ & $3,42^{\mathrm{Aa}}$ & $3,67^{\mathrm{Aa}}$ & $3,67^{\text {Aa }}$ \\
\hline \multirow[t]{2}{*}{16} & $3,67^{\mathrm{Aa}}$ & $3,58^{\mathrm{Aa}}$ & $3,25^{\mathrm{Aa}}$ & $3,34^{\mathrm{Aa}}$ & $3,50^{\mathrm{Aa}}$ & $3,48^{\mathrm{Aa}}$ \\
\hline & \multicolumn{6}{|c|}{ Perda de massa fresca (\%) } \\
\hline 0 & $0,00^{\mathrm{Aa}}$ & $0,00^{\mathrm{Aa}}$ & $0,00^{\mathrm{Aa}}$ & $0,00^{\mathrm{Aa}}$ & $0,00^{\mathrm{Aa}}$ & $0,00^{\mathrm{Aa}}$ \\
\hline 4 & $0,03^{\mathrm{Aa}}$ & $0,02^{\mathrm{Aa}}$ & $0,03^{\mathrm{Aa}}$ & $0,03^{\mathrm{Aa}}$ & $0,03^{\mathrm{Aa}}$ & $0,03^{\mathrm{Aa}}$ \\
\hline 7 & $0,04^{\mathrm{Aa}}$ & $0,04^{\mathrm{Aa}}$ & $0,04^{\mathrm{Aa}}$ & $0,05^{\mathrm{Aa}}$ & $0,06^{\mathrm{Aa}}$ & $0,07^{\mathrm{Aa}}$ \\
\hline 9 & $0,10^{\mathrm{Aa}}$ & $0,10^{\mathrm{Aa}}$ & $0,12^{\mathrm{Aa}}$ & $0,10^{\mathrm{Aa}}$ & $0,10^{\mathrm{Aa}}$ & $0,10^{\mathrm{Aa}}$ \\
\hline 11 & $0,25^{\mathrm{Aa}}$ & $0,29^{\mathrm{Aa}}$ & $0,21^{\mathrm{Aa}}$ & $0,30^{\mathrm{Aa}}$ & $0,27^{\mathrm{Aa}}$ & $0,23^{\mathrm{Aa}}$ \\
\hline 14 & $0,26^{\mathrm{Aa}}$ & $0,28^{\mathrm{Aa}}$ & $0,22^{\mathrm{Aa}}$ & $0,32^{\mathrm{Aa}}$ & $0,20^{\mathrm{Aa}}$ & $0,25^{\mathrm{Aa}}$ \\
\hline \multirow[t]{2}{*}{16} & $0,43^{\mathrm{Aa}}$ & $0,38^{\mathrm{Aa}}$ & $0,46^{\mathrm{Aa}}$ & $0,31^{\mathrm{Aa}}$ & $0,28^{\mathrm{Aa}}$ & $0,39^{\mathrm{Aa}}$ \\
\hline & \multicolumn{6}{|c|}{ Ácido ascórbico (mg ácido ascórbico $\mathrm{kg}^{-1} \mathrm{MF}$ ) } \\
\hline 0 & $211,8^{\mathrm{Aa}}$ & $211,8^{\mathrm{Aa}}$ & $211,8^{\mathrm{Aa}}$ & $211,8^{\mathrm{Aa}}$ & $211,8^{\mathrm{Aa}}$ & $211,8^{\text {Aa }}$ \\
\hline 4 & $216,2^{\mathrm{Aa}}$ & $193,5^{\mathrm{ABab}}$ & $174,5^{\mathrm{BCbc}}$ & $164,3^{\mathrm{Bc}}$ & $161,4^{\mathrm{Cc}}$ & $171,9^{\mathrm{Bbc}}$ \\
\hline 7 & $169,3^{\mathrm{BCa}}$ & $183,8^{\mathrm{BCa}}$ & $171,5^{\mathrm{Ca}}$ & $167,6^{\mathrm{Ba}}$ & $180,8^{\mathrm{BCa}}$ & $190,1^{\mathrm{ABa}}$ \\
\hline 9 & $165,9^{\mathrm{Ca}}$ & $167,5^{\mathrm{Ca}}$ & $163,1^{\mathrm{Ca}}$ & $169,1^{\mathrm{Ba}}$ & $164,7^{\mathrm{Ca}}$ & $166,9^{\mathrm{Ba}}$ \\
\hline 11 & $193,9^{\mathrm{ABa}}$ & $191,7^{\mathrm{ABCa}}$ & $198,7^{\mathrm{ABa}}$ & $203,1^{\mathrm{Aa}}$ & $204,7^{\mathrm{ABa}}$ & $190,2^{\mathrm{ABa}}$ \\
\hline 14 & $210,5^{\mathrm{Aa}}$ & $205,9^{\mathrm{ABa}}$ & $202,7^{\mathrm{Aa}}$ & $199,8^{\mathrm{Aa}}$ & $213,6^{\mathrm{Aa}}$ & $212,3^{\mathrm{Aa}}$ \\
\hline 16 & $196,7^{\mathrm{Aa}}$ & $199,8^{\mathrm{ABa}}$ & $197,7^{\mathrm{ABa}}$ & $198,1^{\mathrm{Aa}}$ & $211,9^{\mathrm{Aa}}$ & $204,5^{\mathrm{Aa}}$ \\
\hline
\end{tabular}

Médias seguidas por mesma letra maiúscula na coluna e minúscula na linha não diferem entre si pelo teste de Tukey $(\mathrm{p} \leq 0,01)$; e BR - armazenamento em balcão refrigerado.

geralmente diminui durante o armazenamento de produtos minimamente processados, mas não foram encontradas referências que explicassem uma redução e posterior aumento em repolho minimamente processado. Provavelmente, esse aumento pode ter ocorrido devido à perda de massa do produto no período transcorrido entre sua retirada das condições de armazenamento e a análise dessa variável, ou devido à heterogeneidade das amostras. Estes resultados diferem do obtido por Silva (2000) e estão de acordo com o observado por Klein (1987).

\section{Conclusões}

A estabilidade do repolho minimamente processado mostrou-se superior na temperatura de $5^{\circ} \mathrm{C}$ quando comparada a do repolho armazenado no balcão refrigerado. A atmosfera modificada ativa não foi efetiva no aumento da vida útil do repolho minimamente processado quando comparada aos outros tratamentos. A embalagem de PEBD foi a mais adequada para o acondicionamento do repolho minimamente processado, quando comparada à embalagem PVC que também apresentou resultados satisfatórios. 


\section{Agradecimentos}

À Fundação de Amparo à Pesquisa do Estado de São Paulo, à Pós-Graduação da Faculdade de Engenharia Agrícola da UNICAMP e à CAPES pelos auxílios concedidos. À White Martins (Praxair Inc.) pelo fornecimento integral das misturas gasosas.

\section{Referências bibliográficas}

ALMEIDA, M. E. M.; NOGUEIRA, J. N. The control of polyphenol oxidase activity in fruits and vegetables: a study of the interactions between the chemical compounds used and heat treatment. Plant Foods for Human Nutrition, v. 47, n. 3, p. 245-256, 1995.

ARAÚJO, J. M. A. Química de alimentos: teoria e prática. Viçosa: UFV, 1999.

CAMERON, A. C.; TALASILA, P. C.; JOLES, D. W. Predicting film permeability needs for modified - atmosphere packaging of lightly processed fruits and vegetables. Horticultural Science, v. 30, n. 1, p. 25-34, 1995.

CANTWELL, M.; SUSLOW, T. V. Postharvest handling systems: minimally processed fruits and vegetables. In: KADER, A. A. (Ed.). Postharvest technology of horticultural crops. Davis: Univ. California; Division of Horticultural and Natural Resources, 2002. p. 445-463.

CHITARRA, M. I. F.; CHITARRA, A. B. Pós-colheita de frutos e hortaliças: fisiologia e manuseio. Lavras: ESAL/FAEPE, 1990. 293 p.

DURIGAN, J. F. Panorama do processamento mínimo de frutas. In: ENCONTRO NACIONAL SOBRE PROCESSAMENTO MÍNIMO DE FRUTAS E HORTALIÇAS, 3., 2004, Viçosa. Resumos... Viçosa: Universidade Federal de Viçosa, 2004. p. 9-12.

ESTAT - SISTEMA PARA ANÁLISES ESTATÍSTICAS. Jaboticabal: Departamento de Ciências Exatas, Universidade Estadual Paulista, [19--].

FANTUZZI, E.; PUSCHMANN, R.; VANETTI, M. C. D. Microbiota contaminante em repolho minimamente processado. Ciência e Tecnologia de Alimentos, v. 24, n. 2, p. 207-211, 2004.

FUJITA, S. et al. Purification and properties of polyphenol oxidase cabbage (Brassica oleracea L.). Journal of Agricultural and Food Chemistry, v. 43, n. 5, p. 1138-1142, 1995.

GÓMEZ-LÓPEZ, V. M. et al. Shelf-life of minimally processed cabbage treated with neutral electrolysed oxidising water and stored under equilibrium modified atmosphere. International Journal of Food Microbiology, v. 17, n. 1, p. 91-98, 2007.

GORNY, J. R. A Summary of CA and MA recomendations for selected fresh-cut fruits. In: INTERNATIONAL CONTROLLED ATMOSPHERE RESEARCH CONFERENCE, 8., 2001, Amsterdam. Proceedings... Amsterdam: [s.n.], 2001.

GOUPY, P. et al. Enzymatic browning of model solutions and apple phenolic extracts by apple poliphenoloxidase. Journal of Food Society, v. 60 , n. 3, p. 479-501, 1995.

INSTITUTO ADOLFO LUTZ. Normas Analíticas do Instituto Adolfo Lutz: métodos químicos e físicos para análise de alimentos. São Paulo, 1985. $371 \mathrm{p}$.

KADER, A. A. Biochemical and physiological basis for effects of controlled and modified atmospheres on fruits and vegetables. Food Technology, v. 40, n. 5, p. 99-104, 1986.

KADER, A. A.; SINGH, R. P.; MANNAPPERUMA, J. D. Technologies to extend the refrigerated shelf life of fresh fruits and vegetables. In: TAUB, I. A.; SINGH, R. P. (Eds.). Food Storage Stability. Boca Raton: CRC Press, 1998. p. 419-434.

KAWANO, S. et al. Cold storage of shredded cabbage. Report of the National Food Research Institute, v. 45, p. 86-91, 1984.
KHAN, A. A.; ROBINSON, D. S. Hydrogen donor specificity of mango isoperoxidases. Food Chemistry, v. 49, n. 4, p. 407-410, 1994.

KLEIN, B. P. Nutritional consequences of minimal processing of fruits and vegetables. Journal of Food Quality, v. 10, n. 3, p. 179-193, 1987.

KLUGE, A. R. et al. Fisiologia e Manejo Pós-Colheita de Frutas de Clima Temperado. Campinas: Editora Rural, 2002. 214 p.

MARTH, E. H. Extended shelf life refrigerated foods: microbiological quality and safety. Food Technology, v. 52, n. 20, p. 57-62, 1998.

MATTIUZ, B. et al. Processamento Mínimo de Uvas de Mesa Sem Semente1. Revista Brasileira de Fruticultura, v. 26, n. 2, p. 226-229, 2004.

NAWA, R. et al. Quality evaluation and preservation of shredded vegetables. 2. Charges in total phenol content and polyphenol oxidase activity in different cultivars of cabbage during storage after shredding. Report National Food Research Institute, v. 50, p. 65-69, 1987.

OLIVEIRA, L. M. et al. Ensaios para Avaliação de Embalagens Plásticas Flexíveis. Campinas: Ed. Centro de Tecnologia de Embalagem - ITAL, 1996. $219 \mathrm{p}$.

RINALDI, M. M.; BENEDETTI, B. C. Influência da Embalagem de polietileno de baixa densidade e da temperatura na conservação do repolho minimamente processado. Engenharia Agrícola, v. 24, n. 2, p. 412-420, 2004.

RINALDI, M. M.; BENEDETTI, B. C.; CALORE, L. Efeito da embalagem e temperatura de armazenamento em repolho minimamente processado. Ciência e Tecnologia de Alimentos, v. 25, n. 3, p. 480-486, 2005.

SAKR, S. et al. Cutting, ageing and expression of plant membrane transporters. Biochimica et Biophysica Acta, v. 1330, n. 2, p. 207-213, 1997.

SARANTÓPOULOS, C. I. G. L. et al. Efeitos da embalagem e da temperatura de estocagem na qualidade de couve minimamente processada. Brazilian Journal of Food Technology, v. 6, n. 2, p. 185-190, 2003.

SCHLIMME, D. V. Marketing lightly processed fruits and vegetables. HortScience, v. 30, n. 1, p. 15-17, 1995.

SILVA, E. O. Fisiologia pós-colheita de repolho (Brassica oleracea var. capitata) minimamente processado. 2000.79 p. Tese (Doutorado em Fisiologia Vegetal) - Universidade Federal de Viçosa, Viçosa, 2000.

TELES, C.S. et al. Evaluación de las alteraciones químicas, físicas y sensoriales en col (Brassica oleraceae, var. acephala) mínimamente procesad conservada sobre atmósfera modificada. Alimentaria: Revista de tecnología e higiene de los alimentos, v. 363, n. 3, p. 112-117, 2005.

VANETTI, M. C. D. Segurança microbiológica em produtos minimamente processados. In: ENCONTRO NACIONAL SOBRE PROCESSAMENTO MÍNIMO DE FRUTAS E HORTALIÇAS, 3., 2004, Viçosa. Palestras... Viçosa: UFV, 2004. p. 30-32.

WHITAKER, J. R.; LEE, C. Y. Recent advances in chemistry of enzymatic browning: an overview. In: LEE, C. Y.; WHITAKER, J. R. (Ed.). Enzymatic browning and its prevention. Washington: American Chemical Society, 1995. p. 2-7.

WILEY, R. C. Minimally processed refrigerated fruits and vegetables. London: Chapman \& Hall, 1994. 357 p.

YANO, M.; SAIJO, R. New preservation method for sheredded cabbage with special reference to nonbrowning cultivar. Journal Japonese Society Cold Preservation Food, v. 13, n. 1, p. 11-15, 1987.

ZAGORY, D. Principles and Practice of Modified Atmosphere Packaging of Horticultural Commodities. In: FARBER, J. M.; DODDS, S. K. L. (Eds.). Principles of Modified-Atmosphere and Sous-Vide Product Packaging. Lancaster: Technomic Publishing Co Inc., 1995. p. 175-204.

ZAGORY, D. Effects of post-processing handling and packaging on microbial populations. Postharvest Biology and Technology, v. 15, n. 3, p. 313-321, 1999. 